\title{
Contribución al Estudio del Sindrome de Ovarios Poliquísticos
}

\author{
Dr. Jorge Amorocho Carreño * \\ Dr. Arturo Aparicio Jaramillo *
}

El propósito de este trabajo es el de aportar algunos datos adicionales al estudio del síndrome denominado "ovarios poliquísticos". En el mismo nombre hay confusión. En efecto, en una época se denominó "ovaritis escleroquística", nombre a todas las luces erróneo, ya que prejuzga de la etiología o por lo menos patogenie inflamatoria de la entidad.Por otro lado, en recientes libros y revistas se encuentra la denominación de "ovarios poliquísticos" en referencia al sindrome de Stein Leventhal.

Hemos querido intentar una diferenciación en la denominación de las dos entidades. En el Sindrome de Stein-Leventhal, hay anatomopatológicamente una marcada esclerosis de la albugínea ovárica, con apariencia lisa exterior, blanca- grisosa, etc. Los quistes múltiples se encuentran únicamente al hacer cortes de la glándula. Esto tal vez haría como más aconsejable el nombre de "ovario esclero-quístico". En la entidad que nos ocupa, el ovario presenta múltiples quistes macroscópicos, e histológicamente puede o no, como sucede en muchos casos, existir esclerosis discreta de la albugínea. Los quistes múltiples hacen saliente sobre la superficie del ovario, haciéndola irregular, y dándole una tonalidad de color completamente distinta. Ello nos inclina a denominar la entidad como 'ovarios poliquísticos".

Para mayor diferenciación de las dos entidades, podremos definir la última como un síndrome hiperestrogénico, caracterizado por trastornos menstruales en la mayor frecuencia de tipo hiper-polimenorréicos, debido a la presencia de múltiples quistes de retención en los ovarios, de etiología variada y de relativa frecuencia, en contraposición con la primera. Síndrome de Stein-Leventhal, cuadro clínico hipoestrogénico, de poca frecuencia, con amenorrea y fenómenos de virilización.

En general podemos decir que es poco lo que se ha estudiado el síndrome de ovarios poliquísticos como entidad global. En la mayoría de los casos se hace alusión a quistes ováricos de reten-

* Del Servicio de Ginecología - Hospital San Juan de Dios 
ción como afección más o menos secundaria dentro de la patología ginecológicas. Es nuestro sentir que el ovario poliquístico es mucho más frecuente y significativo de lo que se juzga en general y que ocupa lugar destacado de las entidades que agrupamos dentro de la denominación de disfuncionales.

\section{MATERIAL}

Hemos tomado cien casos de nuestras pacientes privadas, con el objeto de estudiar el cuadro clínico. Se hicieron pruebas funcionales tales como biopsia endometrial, estudio de citología exfoliativa, metabolismo basal y curvas de Temperatura Basal.

Como la mayoría de las pacientes había sido tratada antes de hacer el estudio, no pudimos seguir un método uniforme, pues la mitad de las pacientes fueron sometidas a cierta rutina de examen y la otra mitad, a un método distinto. Pero en líneas generales puede observarse que el análisis funcional es sensiblemente igual.

Por tratarse de pacientes privadas, en muchas de ellas se omitieron exámenes de Laboratorio costosos, que en rigor hubieran tenido valor especulativo más que directamente práctico para la paciente, exámenes que se hubieran practicado por sistema en un medio hospilatario. El análisis de nuestras pacientes se presenta en los siguientes cuadros estadísticos:

\section{CUADRO № 1 \\ SINTOMATOLOGIA}

Frecuencia

1 - Dolor hipogástrico y $\left.\right|_{0}$ lumbar intermenstrual ... . . 63\%

2 - Dolor abdominal y|o lumbar premenstrual . . . . . 47\%

3 - Mastalgia pre-menstrual . . . . . . . . . . . . . . $73 \%$

4 - Tensión premenstrual . . . . . . . . . . . . . . . $56 \%$

5 - Amenorreas y oligomenorreas . . . . . . . . . . 20\%

6 - Hipomenorreas . . . . . . . . . . . . . . . . . . . . . . . . . $12 \%$

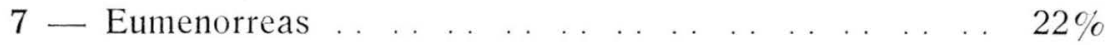

8 - Hipermenorreas . . . . . . . . . . . . . . . . . . . . $36 \%$

9 - Menometrorrogias . . . . . . . . . . . . . . 26\%

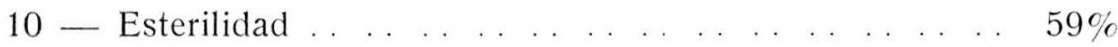

11 - Menalgia . . . . . . . . . . . . . . . . . $74 \%$

12 - Tumefacción ovárica palpable, uni o bilateral . . . . 65\%

Se analizaron también las pruebas funcionales a las cuales habían sido sometidas las pacientes, se presentan en el siguiente cuadro: 
Vol. XI

I - Biopsia endometrial.

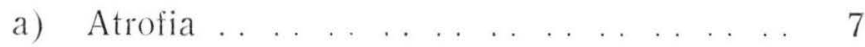

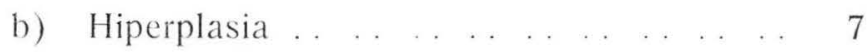

c) Proliferativo persistente . . . .

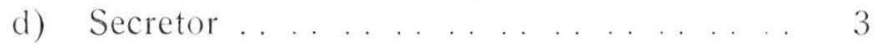

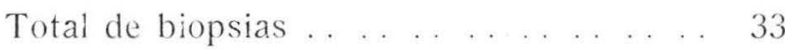

II - Temperatura Basal.

a) Monofásica

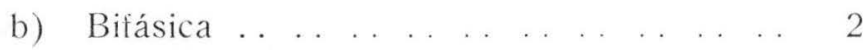

Total de Temperaturas basales . . . . 29

III - Citología vaginal.

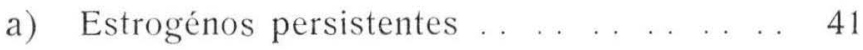

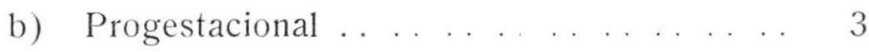

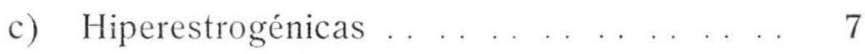

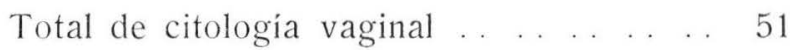

IV - Moco cervical.

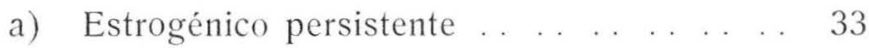

b) Progestacional $\ldots \begin{array}{llllllllll} & \ldots & \ldots & \ldots & \ldots & \ldots & \ldots & \ldots & 2\end{array}$

Total de pruebas sobre moco cervical . . . 55

CARACTERES GENERALES

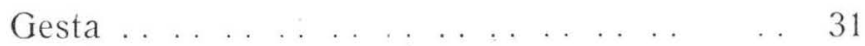

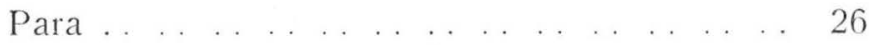

$\begin{array}{llllllllllllll}\text { Abortos } & \ldots & \ldots & \ldots & \ldots & \ldots & \ldots & \ldots & \ldots & \ldots & \ldots & \ldots & 5\end{array}$

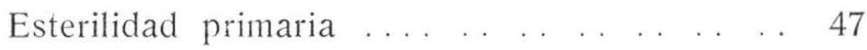

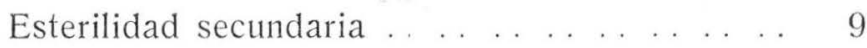




\section{CONCLUSIONES}

Las conclusiones que ha sido posible obtener de estos cuadros, se resumen como sigue:

$1^{\circ}$-Definición

El síndrome de ovarios poliquísticos es un cuadro hiperestrogénico real o relativo constituído por:

a) Hiperpolimenorreas.

b) Anovulación.

c) Síntomas de tensión pre-menstrual.

d) Dolor pélvico y o lumbar premenstrual y $\mid 0$ intermenstrual.

e) Tumefacción ovárica uni o bilateral.

$2^{\circ}$-No debe confundirse con el síndrome de Stein-Leventhal, completamente distinto. Sus caracteres clínicos comparados son:

SINDROME DE OVARIOS POLIQUISTICOS

1-Poli- hiper-meno- metrorra gias: usual.

2-Hipo -oligo- amenorreas: Ocasional y transitorias.

3-Desfeminización: ausente.

4-Tumefacción ovárica no simétrica; muchas veces unilateral o no papable.

5-Anovulación y esterilidad muy frecuentemente secundarias.

6-Presencia de múltiples quistes superficiales.

7-Relativamente frecuente.

\section{STEIN-LEVENTHAL}

1-Poli - hipermeno- metrorragias: ocasional y transitorias.

2-Hipo-oligo- amenorraes: usua les.

3-Desfeminización (hirsutismo, acné, etc.) frecuente $50 \%$.

4) Tumefacción ovárica, tres a cinco veces tamaño ovario normal, BILATERAL; SIMETRICA).

5-Anovulación y esterilidad primarias.

6-Albugínea esclerosa, engrosada, que impide la saliente lobulada de quistes.

7-Relativamente rara. 
$3^{\circ}$ - Los caracteres clínicos del síndrome de ovarios poliquísticos son:

1- Tumefacción ovárica uni o bilateral sensible a la palpación.

2 - Polimenorreas.

3 - Hipermenorreas.

5- Mastalgia premenstrual.

5- Mastalgia premenstrual.

6- Menalgia tipo congestivo o mixto.

7- Dolor pélvico y|o lumbar pre-menstrual y|o intermenstrual.

8- Esterilidad primaria o secundaria por anovulción.

9- Tensión pre-menstrual

$4^{\circ}$ - Los caracteres endocrinos del síndrome son:

1- Hiperfoliculinismo real o relativo:

a) Exceso de estrógenos con hiperplasia de endometrio.

b) Persistencia folicular y anovulación con niveles estrogénicos normales.

c) Ciclos ovulatorios con baja producción de progesterona: el endometrio no pasa de fase secretora inicial.

2- Hipotiroidismo clínico o sub-clínico; en muchas ocasiones función tiroidea normal.

3- Funciones suprarrenales en muchas ocasiones normales.

De lo expuesto, podemos deducir que el criterio diagnóstico seguido en el caso del síndrome de ovarios poliquísticos está basado en los siguientes puntos:

1- Transtornos del ciclo menstrual.

2- Mastalgia y tensión premenstrual.

3- Persistencia estrogénica, demostrada en citología vaginal, moco cervical, temperatura basal y biopsia endometrial.

4- Tumefacción ovárica uni o bilateral.

Expresamente no hemos hecho comentarios respecto a la etiologia, patogenie y tratamiento. 
La etiología permanece aún bastante obscura, o por lo menos parece ser múltiple. En algunos casos se encuentran antecedentes inflamatorios; en otros se puede hallar un disturbio endocrino subclínico, De tal manera que el origen de la lesión parece ser múltiple.

En cuanto se refiere a tratamiento, el que hemos empleado más frecuentemente es el quirúrgico, haciendo resección cuneiforme y extirpando la zona quística. Sinembargo no se nos escapa que éste no es el tratamiento ideal de la entidad. Consideramos que este síndrome es esencialmente funcional y por lo tanto su tratamiento debe orientarse hacia la parte médica. Sobre estos puntos hemos seguido estudiando.

\section{SUMARIO Y CONCLUSIONES}

Hemos presentado nuestras experiencias en cien casos de lo que hemos denominado síndrome de ovarios poliquísticos. Expusimos las razones por las cuales esta debe ser su denominación, dejando la de ovarios escleroquísticos para el síndome de Stein-Leventhal.

En nuestro estudio hemos puesto de relieve la importancia de este síndrome, por su frecuencia y por su significación en la patología ginecológica. Hemos creído dar una visión de conjunto que lo individualiza como entidad.

Hemos estudiado sus características clínicas, y sus modalidades endocrinas.

No hemos contemplado en el presente trabajo el problema referente a etiología, patogenie ni tratamiento, por considerarlos aún en estudio. 